\title{
Las TIC en la UNAN-Managua, crecimiento y desarrollo, periodo 2015-2016
}

MSc. Pedro Aburto Jarquín

Vicerrector Administrativo y de Gestión

UNAN-MANAGUA

paburtoj@unan.edu.ni

DOI: http://dx.doi.org/10.5377/torreon.v6i16.6555

Palabras claves: TIC, SUU-DT, Calidad

"La flexibilización de las instituciones de educación superior para adaptarse a las necesidades de la sociedad actual pasa por la explotación de las tecnologías de la información y la comunicación en los procesos de formación. Pero, al mismo tiempo, ello implica cambios en la concepción de los alumnos-usuarios, cambios en los profesores y cambios administrativos en relación con el diseño y distribución de la enseñanza y con los sistemas de comunicación que la institución establece" (Salinas, 1999).

\section{RESUMEN}

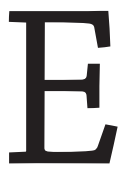

n el presente artículo se expone, a manera de justificación, el Impacto que tienen las TIC a través de la SIU-DT en la UNAN-MANAGUA, impacto en la Docencia, Investigación, Extensión Universitaria e Internacionalización. Se hace una breve descripción de su evolución hasta la actualidad, con el propósito de presentar cómo ha venido dando respuestas de Tecnología Informática a todas las Facultades, Centros de Investigación y Unidades Administrativas.

Estos impactos en ascendencia se deben, precisamente, a toma de decisiones acertadas que se han venido tomando en función de favorecer la Calidad en todos los procesos; por ello, desde el rectorado se ha venido invirtiendo sostenidamente, para mantener también un crecimiento y desarrollo de las TIC, y potenciar el uso y sus aplicaciones académicas y administrativas. 


\section{INTRODUCCIÓN}

Cualquier país, para avanzar en su desarrollo, tiene que invertir en TIC. Las TIC ha intervenido significativamente en el desarrollo cultural, social, económico y en el desarrollo de la tecnología misma, su valor es incalculable. Las empresas, instituciones, las personas, necesitan de estas herramientas para minimizar costos, diversificar el mercado, potenciar la calidad de los procesos, ya no digamos el educativo, que tanto se auxilia de estas herramientas: Data show, Tablet, Celulares, Vídeo conferencias, otros. Nicaragua, como país, avanza en esta ruta, buscando invertir en tecnología para el desarrollo futuro.

Educativamente, las inversiones en tecnología en la UNAN-MANAGUA, cada año han venido creciendo, dado que es una necesidad que los docentes e investigadores demandan para cumplir con mayor calidad su trabajo tanto docente como investigativo. No se puede obviar la importancia de las TIC para el desarrollo de las otras funciones universitarias, como la Extensión, Internacionalización y Gestión propiamente dicho.

En la sociedad del conocimiento, nadie debe dar por entendido que todo lo conoce o todo lo sabe. Cada día las personas aprenden algo nuevo, más aún, hay que tener en cuenta que los conocimientos recibidos en primaria, secundaria y en la universidad misma, no son los necesarios para desempeñarse en la vida social y laboral. Las TIC proporcionan las herramientas complementarias para lograr llegar a ese estadio, de tener un conocimiento actualizado, pero al mismo tiempo muy parcializado. ¿Por qué? Apenas conocemos un poco del área de nuestra especialidad en la cual nos formamos y conocemos menos aquellas otras áreas que por cultura general se quiere documentar o interiorizar algo novedoso. El conocimiento hoy es muy cambiante; lo que ayer era verdad sobre la realidad para determinados fenómenos, horas después ya cambió esa verdad.

Las TIC en la UNAN-MANAGUA han cobrado un giro muy importante y están enfocadas en atender toda aquella demanda estudiantil, que por distintas razones no puede ingresar en los recintos esparcidos por todo el territorio. Ahora, por principio de equidad, será posible virtualmente brindar mayor acceso a la educación superior.

Con la utilización de las TIC se favorece el proceso enseñanza-aprendizaje; estas auxilian los métodos pedagógicos-didácticos que utilizan tanto los docentes para favorecer la enseñanza y el aprendizaje colaborativo, así como el aprendizaje autónomo del estudiante.

Recientemente, la UNAN-MANAGUA, a través de la Dirección de Docencia de Grado, ha informado que evaluarán el currículo aprovechando las TIC. Es importante reconocer que, el uso y potencial de las TIC permite entre otras cosas:

- Acceso a todo tipo de información. 
- Todo tipo de proceso de datos y de manera rápida y fiable.

- Canales de comunicación inmediata, sincrónica y asincrónica, para difundir información y contactar con cualquier persona o institución del mundo.

Las TIC serán el recurso más importante en el presente siglo, para las Universidades, Institutos, Centros de Educación Secundaria y Primaria. Desde el Pre-escolar se va a requerir la cobertura para dar mejor y mayor cumplimiento a uno de los retos del milenio. Muchos niños, niñas, jóvenes y adultos en general, técnicos y profesionales demandarán de la educación virtual. La presencialidad va quedando un poco relegada, la educación del futuro dependerá del uso de las TIC en todas sus modalidades y formas.

La Dirección de Docencia de Grado está desarrollando un proceso de evaluación curricular en sus Facultades y el Instituto Politécnico de la Salud "Luis Felipe Moncada" (POLISAL), con el objetivo de identificar fortalezas y debilidades del Plan de Estudios 2013 de las diferentes carreras que ofrece la UNAN-MANAGUA y así mejorar la calidad de los profesionales que forma.

Para ello, ha realizado talleres de capacitación con los docentes de los diferentes Departamentos sobre el sistema SPSS (Startical Product and Sevice Solutions, conocido también como: Statistical Package for the Social Sciences), un sistema para el manejo y procesamiento de datos que servirá como herramienta para analizar estadísticamente aspectos relacionados al perfil de la carrera, acciones administrativas, uso de la tecnología durante las clases, actividades de extensión social, entre otras.

Otro grupo de docentes de la Facultad de Ciencias, por ejemplo, reciben curso especializado sobre Microsoft para certificarse en estas nuevas tecnologías, otros ya lo recibieron al inicio del año (certificados por la empresa Microsoft), curso a nivel de Maestrías, implementado para especializar al personal docente para optimizar el uso de las TIC en el desarrollo de sus clases.

\section{OBJETIVOS}

1. Presentar a la comunidad universitaria y población en general, el crecimiento y desarrollo de las TIC en la UNAN-MANAGUA.

2. Describir cuáles han sido los impactos de las TIC en Docencia, Investigación y Extensión Universitaria.

3. Resaltar los fondos invertidos en servicios, equipos y medios informáticos en la SIUDT en la UNAN MANAGUA.

\section{ANTECEDENTES}

La Dirección de Sistemas de Información y Desarrollo Tecnológico en UNAN-MANAGUA, tiene su origen a partir del año 2004, conocido en ese momento como Proyecto TIC. A partir de 
ese año, se tiene una nueva concepción y por esto, comenzó a desarrollarse toda la infraestructura de redes y servidores para atender todas las demandas y servicios TIC de las diferentes unidades académicas y administrativas. Prácticamente, se dio una Reingeniería en toda esta unidad o sistema.

La UNAN-MANAGUA contaba, en ese entonces, con máquinas que no tenían la capacidad necesaria para poder gestionar la información automatizada ni poder brindar los servicios demandados en ese momento; únicamente atendían las demandas de la Biblioteca Central del RURD. El personal contratado, principalmente el personal técnico, en el periodo 2005-2016, ha venido formándose y experimentándose en el camino. Hoy, el talento humano de la unidad SIUDT cuenta con personal calificado técnica y profesionalmente. Se cuenta con especialistas en Servidores, en Seguridad informática, en Telemática, Ingenieros electrónicos, todos certificados por Microsoft. El tipo de infraestructura actualmente desarrollada en la UNAN-MANAGUA, es una de la más competitiva, pero además, se han desarrollado Software que han potencializado el avance en tecnología en las Facultades, Centros e Institutos.

De igual manera, los equipos con los cuales se cumple con las demandas de todas las unidades académicas y administrativas, han venido mejorando su capacidad y su calidad. Se nota en las tablas finales donde se refleja la inversión presupuestaria, los equipos y servicios que han permitido contar con las condiciones favorables para atender la demanda de servicios y de la tecnología informática solicitada,

La UNAN-MANAGUA es una de las Universidades Públicas, a nivel nacional, mejor equipada y con los equipos e infraestructura mejor desarrollados (Gerardo Paz, Técnico del SIUDT).

\section{Transición y Desarrollo de las TIC en la UNAN-Managua}

Justin García, técnico del SIU-DT con tres años y medio de servicio, y Derman Zepeda, Director actual, exponen que el Registro Académico Estudiantil tiene aplicaciones TIC desde los años 80. En la década de los 90, la UNAN-MANAGUA invirtió más en equipos informáticos, dotando a todas las Facultades e Instituto Politécnico de la Salud (IPS). Se construyeron los primeros laboratorios de computación para las carreras relacionadas. Es a partir del año 95 que se introducen las redes locales (ISDN)루 a finales de la década, la UNAN-MANAGUA, tenía interconectada a Internet una buena parte de sus equipos, principalmente las oficinas de las direcciones y los laboratorios de computación. En el 2000 se instaló el primer NODO con red telefónica interna. Este nodo también brindaba todo tipo de servicios relacionado a las instancias superiores, las Facultades y Centros e Institutos de Investigación.

1. Red Digital de Servicios Integrados. Disponible en: http://informatica.uv.es/iiguia/AER/Tema8.pdf 
En el 2005 se desarrolló por primera vez una red local que funcionaba para toda la Universidad. Todos los pabellones tenían acceso a Internet. Los docentes tenían ya disposición para utilizar los medios técnicos en el aula de clases de manera directa.

Entre otros servicios que brindaba esta unidad estaban, por ejemplo:

- Grupo cerrado de usuarios

- Identificación del usuario llamante

- Restricción de la identificación del usuario llamante

- Identificación de usuario conectado

- Restricción de la identificación de usuario conectado

- Identificación de llamada en espera

- Marcación directa de extensiones

- Conferencia a tres

- Desvío de llamadas

- Transferencia de llamadas

- Información de tarificación y consumo

De 2005 a 2016, la inversión en medios y equipos para la Dirección del SIUDT y las Facultades, ha sido tanto en equipos y medios, como en su crecimiento y desarrollo de Sistemas de Información. Solo en los dos últimos años, 2015 y 2016, se han invertido casi C\$37 millones de córdobas, (ver anexos), situación ventajosa para la universidad, por estar dotada de los medios, infraestructura y personal técnico y profesional capacitado para brindar los servicios a través de los distintos Sistemas de Información actualmente desarrollados.

Hoy, la universidad es la encargada de la red de datos y comunicación que comprende las redes ubicadas en las FAREM, Ciencias Económicas, Recinto Ricardo Morales Avilés, Centro de Investigación de Recursos Acuáticos, Recinto Universitario Rubén Darío, y es la unidad creadora de los distintos Sistemas de Información. Además, se han creado espacios especiales para los estudiantes "KIOSKOS" con los enlaces Wifi en todos los espacios del Recinto Universitario Rubén Darío, igual sucede en todas las FAREM (se ha invertido C $\$ 3$ millones de córdobas), cabe destacar también, que todas las Facultades cuentan con un espacio para videoconferencias con las condiciones tecnológicas necesarias para conectarse en cualquier momento, con cualquier institución o universidad nacional o internacional. 
Las Tecnologías de la Información y la Comunicación, también conocidas como TIC ${ }^{2}$, "son el conjunto de tecnologías desarrolladas para gestionar información y enviarla de un lugar a otro. Por tal razón, el uso de las redes de telecomunicaciones en la enseñanza es aplicada en la educación a distancia. Cabe destacar que, las instituciones universitarias se encuentran en transición. Por esto, la educación a distancia se puede tornar un poco fuerte, por el hecho de que en ocasiones pueden resultar algunos problemas, en cuanto a la comunicación de las redes, pero ahora no solo se dan a distancia sino, que también su uso se aplica en la enseñanza presencial, mostrando beneficios".

Juan Navas, con casi 15 años de servicios en esta unidad, menciona la necesidad de Certificación del SIU-DT, "ya tenemos las condiciones creadas y para ser aún más competitivos, es necesario la certificación".

Los entrevistados consideran importante capacitar (y ellos están dispuestos a hacerlo) a docentes e investigadores sobre las TIC y sus potencialidades como herramienta informática, su desconocimiento implica mal uso de los medios y equipos, pero, sobre todo, pierden la oportunidad que prestan estas herramientas para optimizar la calidad de sus clases y el desarrollo de aprendizajes y capacidades de los estudiantes. Finalmente, sugieren establecer contacto con el GRUN (Gobierno de Reconciliación y Unidad Nacional), para optimizar el uso de la Banda Ancha recientemente introducida en Nicaragua.

\section{Servicios que ofrece TIC UNAN-MANAGUA, FAREM-CHONTALES}

1. Capacitación en Moodle y herramientas tecnológicas para la educación a docentes de FAREM-CHONTALES

2. Adquisición de equipos multimedia para el apoyo de trabajo docente

3. Cursos en línea y Videoconferencias

4. Elaboración de material impreso y digital de proyección Universitaria:
a. Documentales
b. Spot Publicitarios
c. Banner
d. Revistas
e. Dípticos y Trípticos

5. Creación de Aplicaciones y Sistemas Administrativos, Desarrollo de sitios Web

2. http://aprendeenlinea.udea.edu.co/lms/investigacion/mod/page/view.php?id=3118 

a. Tecnologías HTML5, Angular, React, Javascript, NodeJS, entre otros
b. Sistemas de Gestión Académica y Administrativa

6. Cable estructurado y configuración de servidores
a. DHCP, PROXY, cableado de redes
b. Conformar área TIC
c. Área de desarrollo de software, aplicaciones Web para empresas
d. Servidor dedicado para realizar pruebas, desarrollo y producción
e. Equipos: Cámaras HD, MICROFONOS profesionales

\section{Servicios que puede ofrecer TIC UNAN-MANAGUA, FAREM-CHONTALES}

1. Cursos y Capacitaciones semipresenciales y presenciales, dirigidos al sector social y educativo orientado al uso de las TIC como herramienta necesaria en la vida diaria

2. Mantenimiento de hardware y software a equipos informáticos

3. Servicios Multimedia (videos, imágenes, programas de radios y televisión)

4. Diseño de página Web y Sistemas de Información

5. Alojamiento Web

6. Diseño e instalación de redes

7. Asesoramiento técnico

8. Grabación de grupos de música

9. Carnetización a empresas e instituciones

10. Instalación y configuración de servidores

11. Asesoría técnica a municipalidades

\section{Impacto de las TIC en la UNAN- MANAGUA}

El impacto obtenido por las TIC, después de invertir más de C $\$ 37$ millones de córdobas, son incalculables. Se trata de impactos intangibles, además, de mejorar el proceso enseñanzaaprendizaje. Las TIC han cambiado la cultura institucional y de todos sus miembros; son impactos, económicos, por ejemplo, se invierten aproximadamente en este periodo la cantidad

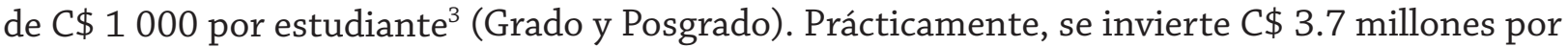
facultad, pero el impacto en el aprendizaje ¿cómo medirlo? El impacto del valor agregado para la universidad ¿cómo cuantificarlo? Las capacidades, habilidades y destrezas desarrolladas por 
los miembros que usan las TIC ¿cómo cuantificarlas? ¿Cómo medir el volumen de información disponible y acumulada por la sociedad del conocimiento?

Cabe resaltar que, con fondos estatales, cada Facultad, Centro e Instituto invierte año con año en tecnología. Cada año las distintas Facultades, Centros e Institutos gestionan otras formas de financiar las TIC. Medicina, Optometría, el POLISAL, LAF-RAM, entre otras unidades académicas, sistemáticamente intercambian conocimientos, tecnología, transferencia tecnológica, equipos médicos.

El proceso de la investigación científica se ve fortalecido también con el uso y aplicación de las TIC de aquellos investigadores que tiene conocimiento y la aplican en sus tesis, desde la presentación y en todo su contenido, nada mejor que usar Ofimática para dar realce y estética en su presentación.

En los estudios tanto cualitativos, como cuantitativos, se hace uso de diferentes programas informáticos para el uso de estadística descriptiva, como la estadística inferencial. Esta última utilizando el paquete estadístico: SPSS (Statistical Product and Service Solutions). Actualmente, la mayoría de las investigaciones son de carácter mixto, por lo que los investigadores usan distintas fuentes, blogs, sitios Web; buscan repositorios de revistas indexadas y de impacto para fundamentar su tesis. Las TIC prestan herramientas tecnológicas a los innovadores, quienes hacen modelos de simulación de diferentes prototipos.

Es más, muchos trabajos de investigación de UNAN-MANAGUA se realizan con el propósito de determinar y delimitar qué tanto uso y aplicaciones hacen los docentes durante el proceso enseñanza-aprendizaje. Esto será un insumo importante para continuar tomando decisiones acertadas en esta materia.

Con esas herramientas se maneja una gran cantidad de datos, los que, procesados correctamente, permiten tomar mejores decisiones para el docente, estudiante, el colaborador, el comerciante, el industrial, el Rector (a), para toda la sociedad en su conjunto.

Internet facilita instrumentos para mejorar los canales de información y comunicación tradicionales de la enseñanza a distancia, permitiendo a los estudiantes un mayor control de su trabajo y al profesorado, un mejor seguimiento de las actividades que realizan los estudiantes.

En el siglo XVIII, auge de la Radio y la TV, el estudiante, por ejemplo, recibía y escuchaba lo que estos medios difundían. Obligadamente tenía que estar pendiente de algunos conocimientos. En la actualidad es lo contrario, con la Internet, por ejemplo, se escucha y estudia lo que se quiere y en cualquier momento se gestiona la información que interesa, además, Internet reduce la necesidad de infraestructura para desarrollar el proceso enseñanza-aprendizaje y a menor costo. La comunicación es versátil, mediante el E-mail, el chat, por teléfono móvil, la Tablet, 
el ordenador, se reduce distancia y tiempo. Actualmente se adquiere un producto vía Internet, se hace una transacción vía billetera móvil. Así se hace, por ejemplo, la matrícula en UNANMANAGUA, al mismo tiempo que los estudiantes gestionan una serie de información que antes era un problema: imprimir las calificaciones del semestre desde un "cyber" (negocio que vende servicios TIC) en cualquier parte de Nicaragua, recibir su beca o estipendio económico vía tarjeta de débito bancaria, conectarse con su docente vía Teléfono móvil, por correo, por cualquier red social.

Las TIC garantizan facilidades administrativas para los docentes: obtención de listas de los estudiantes, envío provisional de actas de calificaciones. Facilidades administrativas para los estudiantes: consultar sus calificaciones y realizar determinados trámites burocráticos y otros. Se pueden facilitar los enlaces a otras páginas Web de la universidad (o de otras instituciones) que pueden ofrecer información y servicios de interés de los estudiantes. Hoy los servicios de videoconferencia son muy comunes para intercambiar conocimientos, técnicas, información y comunicación intra e inter universidades. Además, se ahorran recursos económicos y de tiempo, al trasladarse las personas desde la facultad de origen hacia Managua.

A nivel nacional, el servicio de telefonía ha venido creciendo significativamente, así, por ejemplo, entre el 2010 y 2015 se ha duplicado la demanda. De acuerdo con el documento, en 2008, en Nicaragua había apenas 19280 perfiles de Facebook. Sin embargo, en ocho años, el número de cuentas ha crecido hasta en 77,8 veces.

Para Manuel Díaz, gerente de Listo Marketing, "estos números no solo se refieren a habitantes en Nicaragua, sino gente que puede vivir en otro país, pero es nicaragüense o viceversa, un extranjero que en algún momento puso residencia en Nicaragua".

Por otro lado, otro factor que incide en este enorme crecimiento es que muchos usuarios que no tienen acceso constante a Internet suelen tener más de una cuenta en esta red social. " $E l$ escenario más común es que tuvo acceso a una computadora con Internet, creó su cuenta. Tuvo acceso tiempo después, pero creó otra cuenta porque no recuerda la clave ni el correo", agregó Díaz (Metro de Nicaragua, Julio 13, 2015. Ver anexo Usuarios).

Podrán ver en los anexos que, hay diversas empresas dedicadas a la venta de servicios TIC que tienen un impacto socio-económico en el país. Al mismo tiempo, proporciona una caracterización de hacia dónde vamos y cómo estamos hoy en TIC.

Esta es una oportunidad que tienen los docentes para potenciar el uso del celular. En las aulas universitarias, casi el $90 \%$ de los estudiantes portan celulares con servicios de Internet, otro buen porcentaje lo utiliza en los pasillos donde se tiene conexión Wifi. Si bien es cierto que 
las PC y las Tablet son usadas para hacer tareas asignadas, también es cierto que son usadas para conectarse en las redes sociales.

Con el uso de las TIC, se deben crear plataformas amigables con los aparatos, dentro del aula y fuera de la misma. Los estudiantes deben utilizar estos aparatos para recibir, intercambiar, bajar, transferir información para él, para el tutor y para sus compañeros de trabajo. Esto tendría sus ventajas en el sentido de garantizar una buena sesión de clase con una mejor disciplina estudiantil. Se evita que se conecten por un rato a las redes sociales, que pueden ser improductivas para el mismo estudiante.

Hay un impacto cultural con el uso de la tecnología, pues facilita el uso de las TIC desde todo punto de vista y desde distintas formas y circunstancias. En este siglo, en el que han proliferado las TIC, se tiene aún profesores ermitaños en tecnología, no aceptan la versatilidad de estas, no les gusta capacitarse en este sentido. Debe romperse este paradigma y ¡hay que comenzar ya!

Desde el punto de vista social, el uso social de la tecnología y su acceso desde la universidad es un impacto social, se da mayor sociabilidad entre los colectivos de trabajo, tanto estudiantiles como de docentes y administrativos; se fomenta la comunicación, se aumentan los contactos y los conocimientos. En la actualidad, en muchos parques del país hay Wifi disponible para la sociedad, principalmente para los jóvenes nicaragüenses. A partir del año 2017, el móvil es un instrumento importantísimo para todos aquellos estudiantes que quieran estudiar en la Universidad Abierta en Línea desde cualquier zona remota del país: un nuevo paradigma cultural.

\section{REFERENCIAS BIBLIOGRÁFICAS}

(s.a.). (s.f.). Red Digital de Servicios Integrados.

Disponible en: http://informatica.uv.es/ iiguia/AER/Tema8.pdf

Aprende en línea. (2014). Plataforma académica para la investigación. Las TIC como apoyo a la educación. Tomado de: http://aprendeenlinea.udea.edu.co/lms/ investigacion/mod/page/view.php?id

Blanco, Benjamín. (2015). El Nuevo Diario. Managua.

Pere Marqués, Graells. (2001). Algunas notas sobre el impacto de las TIC en la Universidad.
Universidad Autónoma de Barcelona. Disponible en: http://dewey. uab.es./ pmarques

Salinas, José. (2002). Las TIC como Medio para una nueva universidad. Congreso Internacional Docencia Universitaria e Innovación. Tarragona. Disponible en: http://www.cidui2016/cidui

Zepeda, Derman. (2016). Sistema de Información Universitaria y Desarrollo Tecnológico. SIUDT.UNAN-MANAGUA. 


\section{ANEXOS}

Tabla 1. Inversiones en TIC, UNAN-Managua 2015-2016

\begin{tabular}{|c|c|c|}
\hline No & Nombre de los Procesos 2016 & Presupuestado \\
\hline 1 & Licenciamiento para el centro de datos de la UNAN - Managua & $C \$ 480,000.00$ \\
\hline 2 & $\begin{array}{l}\text { Chasis de servidor con capacidad hasta } 8 \text { servidores blades, } 2 \\
\text { servidores blades instalados }\end{array}$ & $C \$ 1,600,000.00$ \\
\hline 3 & $\begin{array}{l}\text { Hardware y partes para el centro de datos de la UNAN - } \\
\text { Managua }\end{array}$ & $C \$ 1,615,000.00$ \\
\hline \multirow{3}{*}{4} & Servicio de remodelación de oficina SIUDT & $C \$ 245,000.00$ \\
\hline & $\begin{array}{l}\text { Adquisición de Materiales Eléctricos para el Centro de Datos } \\
\text { de la UNAN-Managua }\end{array}$ & $C \$ 160,000.00$ \\
\hline & Servicio de suministro de muebles para la oficina del SIUDT & $C \$ 307,500.00$ \\
\hline \multirow{3}{*}{5} & $\begin{array}{l}\text { Dispositivos activos y accesorios audiovisuales } \\
\text { complementarios para la red y sistema de streaming de la } \\
\text { UNAN-Managua }\end{array}$ & $C \$ 270,000.00$ \\
\hline & $\begin{array}{l}\text { Materiales y herramientas para el mantenimiento de la red de } \\
\text { fibra y cobre de la UNAN-Managua }\end{array}$ & $C \$ 300,000.00$ \\
\hline & $\begin{array}{l}\text { Servicios de soporte y mantenimiento para dispositivos de } \\
\text { alimentación y protección eléctrica de la UNAN-Managua }\end{array}$ & $C \$ 230,000.00$ \\
\hline 6 & $\begin{array}{l}\text { Adquisición de equipos para red inalámbrica de la UNAN } \\
\text { Managua }\end{array}$ & $C \$ 2,000,000.00$ \\
\hline 7 & Cableados estructurados para el RURD y FAREM-Carazo & $C \$ 650,000.00$ \\
\hline 8 & $\begin{array}{l}\text { Enlaces de datos e Internet para la UNAN - Managua (Pago } \\
\text { Anual) }\end{array}$ & $C \$ 4,000,000.00$ \\
\hline 9 & $\begin{array}{l}\text { Adquisición de Suscripción para Licenciamiento Microsoft } \\
\text { Desktop Education para (doscientos cincuenta) usuarios. } \\
\text { Dsktp Edu ALNG Lic SAPk OLV E } 1 Y \text { Acdmc Ent Cantidad: } 250 \\
\text { usuarios. Open Value Subscription. }\end{array}$ & $C \$ 500,000.00$ \\
\hline 10 & Licenciamiento de Antivirus & $C \$ 500,000.00$ \\
\hline 11 & $\begin{array}{l}\text { Adquisición de licencias para Certificaciones Microsoft - } 250 \\
\text { MTA; } 500 \text { MOS - } 250 \text { MCE }\end{array}$ & $C \$ 285,000.00$ \\
\hline 12 & Extensión de Contrato de Internet (1 mes) & $C \$ 41,030.80$ \\
\hline \multirow[t]{2}{*}{13} & Materiales de Eléctricos, Datacenter & $C \$ 2,319.55$ \\
\hline & Total invertido & C $\$ 13,185,850.35$ \\
\hline
\end{tabular}


Tabla 2. Inversión TIC 2016

\begin{tabular}{|c|c|c|}
\hline No & Nombre de los Procesos 2015 & Presupuestado \\
\hline 1 & $\begin{array}{l}\text { Adquisición de materiales y herramientas para mantenimiento del } \\
\text { cableado de fibra y cobre del RURD }\end{array}$ & $C \$ 124,279.86$ \\
\hline 2 & $\begin{array}{l}\text { Adquisición de disco duros para SAN y NAS para el Centro de datos } \\
\text { de la UNAN - Managua }\end{array}$ & $C \$ 293,702.80$ \\
\hline 3 & $\begin{array}{l}\text { Adquisición de licenciamiento de software para el Centro de datos de } \\
\text { la UNAN - Managua }\end{array}$ & $C \$ 449,733.13$ \\
\hline 4 & Adquisición de dispositivos activos para la UNAN - Managua & $C \$ 448,236.86$ \\
\hline 5 & $\begin{array}{l}\text { Adquisición de servidor tipo rack para ampliar capacidades del cluster } \\
\text { de la UNAN - Managua }\end{array}$ & $C \$ 396,750.00$ \\
\hline 6 & $\begin{array}{l}\text { Adquisición de } 2 \text { kit de memoria de } 128 \text { GB para servidores DELL } \\
\text { R72, servitag 1er server 5LHRV12; servitag 2do server: 2475FX1 del } \\
\text { RURD }\end{array}$ & $C \$ 108,640.00$ \\
\hline 7 & $\begin{array}{l}\text { Adquisición e instalación de infraestructura de red inalámbrica del } \\
\text { RURD /UNAN (Segunda etapa) }\end{array}$ & $C \$ 500,000.00$ \\
\hline 8 & $\begin{array}{l}\text { Adquisición de dispositivos activos para SAN y de acceso para el } \\
\text { RURD/UNAN Managua }\end{array}$ & $C \$ 500,000.00$ \\
\hline 9 & $\begin{array}{l}\text { Compra de aire acondicionado de precisión inrow de 33,000 BTU y } \\
\text { servicio de instalación par el centro de datos del RURD }\end{array}$ & $C \$ 250,000.00$ \\
\hline 10 & Fuente de Poder para Equipo Cisco 4500 & $C \$ 100,000.00$ \\
\hline 11 & $\begin{array}{l}\text { Adquisición de dispositivos y servicios de implementación para } \\
\text { sistemas de telefonía IP de la UNAN Managua }\end{array}$ & $C \$ 494,809.23$ \\
\hline 12 & $\begin{array}{l}\text { Adquisición e instalación de infraestructura de red inalámbrica del } \\
\text { RURD /UNAN (Primera etapa) }\end{array}$ & $C \$ 273,507.31$ \\
\hline 13 & Adquisición de cableado estructurado para la UNAN - Managua & $C \$ 489,623.41$ \\
\hline 14 & $\begin{array}{l}\text { Salas de videoconferencias para la Facultad de Ciencias e Ingenierías, } \\
\text { Educación e Idiomas, Humanidades y Ciencias Médicas, IPS } \\
\text { (POLISAL) de la UNAN Managua }\end{array}$ & $C \$ 2,000,000.00$ \\
\hline 15 & Adquisición de cableado estructurado para FAREM Carazo & $C \$ 270,020.00$ \\
\hline 16 & Servicio de enlaces de Internet y Datos para la UNAN Managua & $C \$ 3,250,551.50$ \\
\hline 17 & Adquisición de 2500 licencias de antivirus para la UNAN Managua & $C \$ 463,456.00$ \\
\hline 18 & Certificaciones Microsoft & C\$ 98,000.00 \\
\hline 19 & $\begin{array}{l}\text { Mantenimiento a las instalaciones del centro de datos del RURD/ } \\
\text { UNAN Managua }\end{array}$ & $C \$ 400,000.00$ \\
\hline 20 & $\begin{array}{l}\text { Sistema de transmisión streaming en vivo desde los auditorios del } \\
\text { RURD/ UNAN - Managua }\end{array}$ & $C \$ 191,076.13$ \\
\hline & Total invertido & $\mathrm{C} \$ 11,102,386.23$ \\
\hline
\end{tabular}


Tabla 3. Proyectos TIC actuales en las Facultades Regionales de Carazo, Chontales, Estelí y Matagalpa

\begin{tabular}{|c|c|}
\hline No. & Nombre de proyecto \\
\hline 1 & Acondicionamiento Acústico e Iluminación de USAV FAREM-Carazo \\
\hline 2 & $\begin{array}{l}\text { Inserción de fibra Óptica de la empresa Claro para brindar servicios de Internet con } \\
\text { ancho de banda de } 40 \text { megas y } 20 \text { megas de datos }\end{array}$ \\
\hline 3 & $\begin{array}{l}\text { Cambio de fibra óptica desde el área de red ubicado en edifico Torreón Universitario, } \\
\text { Posgrado y Laboratorios }\end{array}$ \\
\hline 4 & $\begin{array}{l}\text { Redundancia de Fibra Óptica desde el Área de redes, Biblioteca, Juan Sánchez y } \\
\text { Laboratorio } 3\end{array}$ \\
\hline 5 & $\begin{array}{l}\text { Inserción de la fibra óptica de la empresa claro para llevar servicios de datos e } \\
\text { internet en el Hospital Escuela Regional Santiago }\end{array}$ \\
\hline 6 & $\begin{array}{l}\text { Instalación de un tramo de fibra óptica en el edificio Augusto C. Sandino para llevar } \\
\text { servicio internet a través de datos }\end{array}$ \\
\hline 7 & Adquisición de respaldo eléctrico para 6 horas \\
\hline 8 & Desarrollo de Plataforma Virtual para Cursos MOOC \\
\hline 9 & Capacitaciones en Micro Robótica Educativa \\
\hline 10 & Programa de Capacitaciones para el desarrollo de competencias para el personal TIC \\
\hline 11 & Ejecución de Diplomado en Educación Musical \\
\hline 12 & Ejecución de Diplomado en Producción Musical \\
\hline 13 & $\begin{array}{l}\text { Adquisición de Equipos para el área del USAV (NAS de } 18 \text { TB para almacenamiento } \\
\text { de los materiales generados) y Capturadora de Video y Streaming }\end{array}$ \\
\hline 14 & Adquisición de 5 cañones inteligentes. \\
\hline 15 & Adquisición de sala de Video Conferencia totalmente equipada \\
\hline
\end{tabular}




\section{USUARIOS}

El promedio de crecimiento del servicio de telefonía celular en Nicaragua es de $743 \mathrm{mil}$ 615 nuevos suscriptores cada año.

Líneas celulares desde año 2010 al 2015
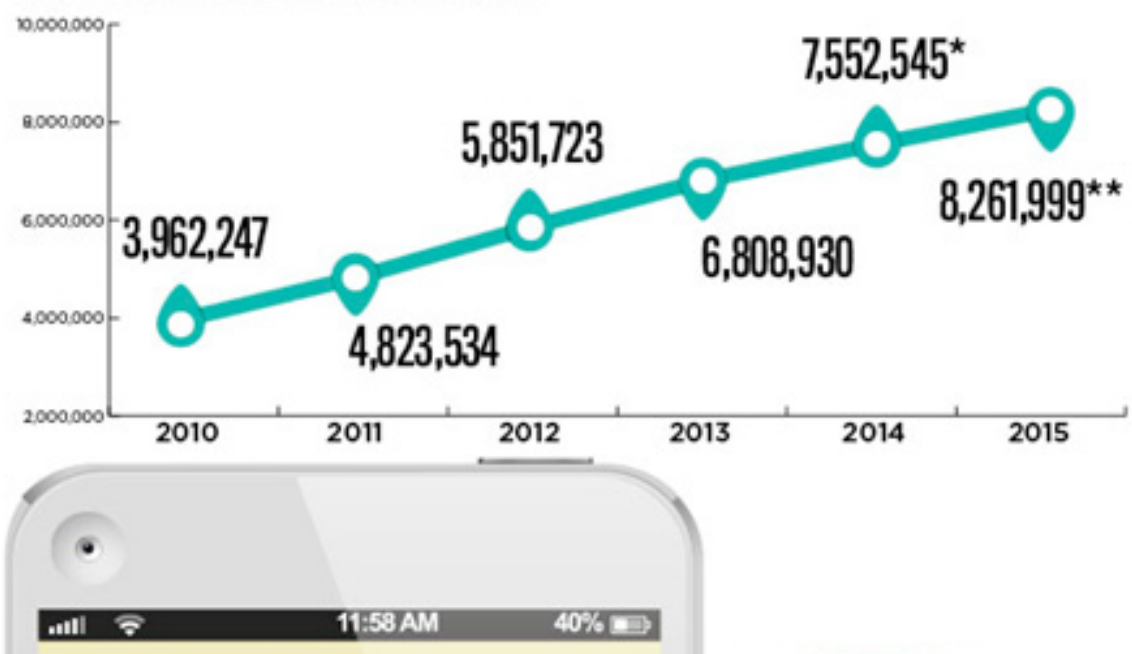

Distribución del parque de smartphones por sistema operativo

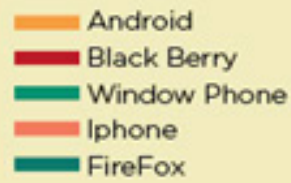




\section{PERFIL GENERAL DE COMPAÑÍAS TIC EN NICARAGUA}

Es difícil determinar cuántas compañías componen el sector de TIC en Nicaragua. La razón principal es la falta de estadíst ica actualizada pública y privada. Sin embargo, aunque el sector TIC de Nicaragua es joven y poco es tructurado, la evidencia indica que la convergencia inalámbrica y fija ha transformado la dinámica con la que las empres as se relacionan con sus clientes. Las entrevistas de este estudio indican que existen unas 88 compañías que trabajan en diferentes especializaciones del sector. El diagrama a continuación muestra su distribución.

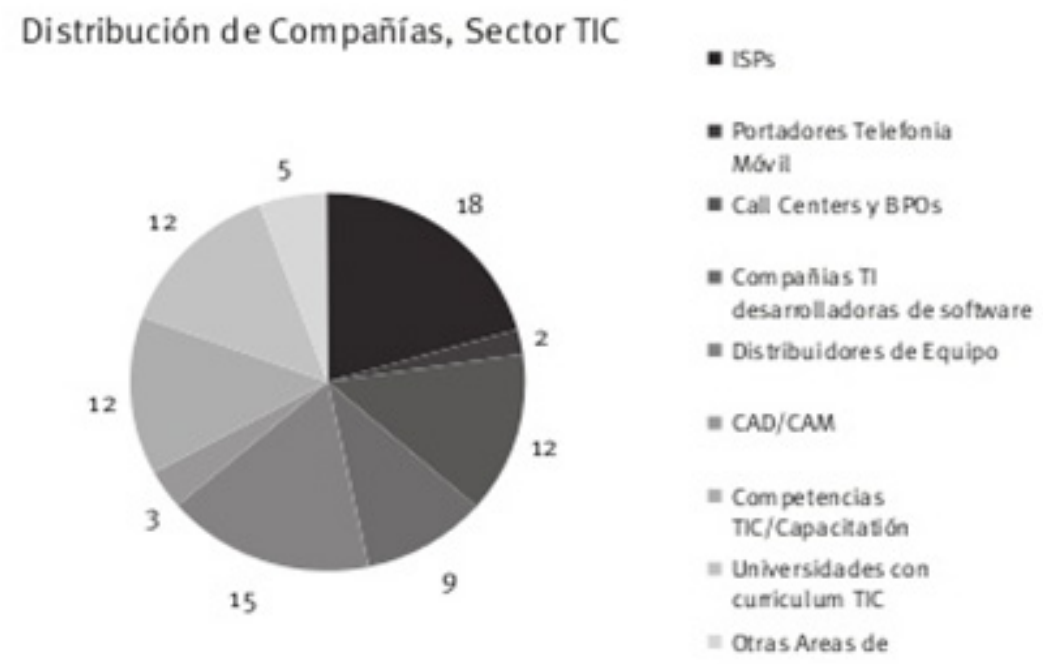

La cartera de clientes de negocios del sector TIC se divide en el sector privado (60-80\%) y el gobierno (20-40\%). El año pasado, las firmas obtuvieron solamente $25 \%$ de los contratos del gobierno, mientras que el $75 \%$ fue otorgado a compañías extranjeras. El sector de TIC, según estimados de ProNic aragua y datos del Banco Central, ha crecido un $17 \%$ anual por cuatro años consecutivos. En 2005 . los réditos de ventas sumaron U\$12.5 millones, mientras que en 2008 sumó US\$ 33 millones. De esos, U\$15 millones fueron generados por el sector de BPOs y Contact Centers.

\subsection{Organizacionestic}

Aunque no hay una organización representativa de todos los intereses del sector TIC en Nicaragua, vale la pena mencionar que se han realizado un buen número de iniciativas para apoyar al sector. EI Consejo Nicaragũense de Ciencias y Tecnología (CONICYT) y el Ministerio de Desarrollo, Indus tria y Comercio, han colaborado con el Banco Mundial y la Agencia Sueca para el Desarrollo Internacional (ASDI) en la elaboración de estrategias nacionales TIC. Actualmente, hay una iniciativa para organizar una Cámara de Comercio TIC, en coordinación con el Consejo Superior de la Empresa Privada (COSEP). Has ta la fecha, la mayoría del trabajo gremial se ha canalizado a través de la Cámara de Industrias y la Cámara de Comercio.

Existe también la Asociación de Internet de Nicaragua (AIN), fundada en el 2002. Sin fines de lucro, existe para unific ar y coordinara los proveedores de servicio de Intemet, y a todas aquellas ins tituciones académicas cuyos esfuerzos son relacionados. Además, ex is te un capítulo local en Nicaragua de la Asociación Internacional de Profesionales de Outsourcing (IAOP - International Association of Outsourcing Professionals), que aglomera a los más grandes proveedores de servicios de Call Centers y BPOs.

Figura 2. Perfil general de compañías TIC en Nicaragua Fuente: Documento "Oportunidades de negocio en el sector de las Tecnologías de Información y Comunicaciones" publicado por Ministerio de Asuntos Exteriores de Dinamarca (pág. 9) 